\title{
MANIPULATION OF LIQUID SPREADING ON ASYMMETRIC NANOSTRUCTURED SURFACES
}

\author{
K.-H. Chu ${ }^{l *}$, R. Xiao ${ }^{l}$, M.E. Alf $f^{2}$, K.K. Gleason ${ }^{2}$ and E. N. Wang ${ }^{1}$ \\ ${ }^{1}$ Department of Mechanical Engineering, and ${ }^{2}$ Department of Chemical Engineering, \\ Massachusetts Institute of Technology, Cambridge, Massachusetts, USA
}

\begin{abstract}
We demonstrate the manipulation of liquid spreading on asymmetric nanostructured surfaces using electrowetting. Asymmetric nanopillar arrays were fabricated with diameters of 500 to $750 \mathrm{~nm}$ and deflection angles of 3 to 52 degrees using deep reactive ion etching and angled metal deposition process on silicon. Droplets can achieve uni-directional, asymmetric, and nearly symmetric spreading behaviors with various applied electric potentials on asymmetric nanostructured surfaces. A theory based on an energy argument is also developed to predict the spreading behaviors and shows excellent agreement with experimental results. This work shows that we can manipulate liquid spreading in a single direction on-demand, which suggests new opportunities to tailor advanced nanostructures to achieve active control of complex flow patterns for various microfluidic and thermal applications.
\end{abstract}

\section{INTRODUCTION}

The manipulation of liquid spreading is important for a broad range of microfluidic, biological, and thermal management applications [1-3]. Engineered surfaces have received significant interest to enable control of liquid behavior, such as varying the liquid film thickness [4] and the final wetted shape [5]. By utilizing groove geometries and patterned surface chemistries, elongated droplets with distinct contact angles in the orthogonal directions have also been achieved [6,7]. In all of the previous studies, however, the spreading behavior on these engineered surfaces is symmetric [4-7]. Furthermore, dynamic control of spreading behavior has not yet been demonstrated.

In this work, we present the design of asymmetric nanostructures that can not only be used to achieve uni-directional spreading [8], but also to actively control the directionality of liquid spreading with applied electric potentials. The asymmetric nanopillars can introduce energy barriers on more than one axis, which allows the liquid to propagate in a single preferred direction and pin in all others. However, with the application of electric potential, the spreading directionalities can change depending on the magnitude of potential difference by decreasing the energy barriers in different directions.

\section{FABRICATION}

Asymmetric nanopillars were fabricated using MEMS processing on silicon with diameters ranging from 500 to $750 \mathrm{~nm}$, spacings of $3.5 \mu \mathrm{m}$, and heights of 9 to $10 \mu \mathrm{m}$. The pillars were patterned using projection lithography, and etched in silicon with deep reactive ion etching (DRIE). A thin gold film was subsequently deposited onto one side of the pillars with an electron beam evaporator. The residual stress between the two materials caused the pillars to deflect. The thickness of the gold film controlled the deflection angle.

Fig. 1 shows scanning electron micrographs (SEMs) of three representative asymmetric nanopillar arrays with deflection angles, $\varphi$, (as defined in the Fig. 1 inset) ranging from $7^{\circ}-25^{\circ}$. A Cartesian coordinate system is defined for convenience, as shown in Fig. 1, where the pillars deflect in the positive $\mathrm{X}(+\mathrm{X})$ direction. After the fabrication, we conformally coated the nanopillars with a polymer

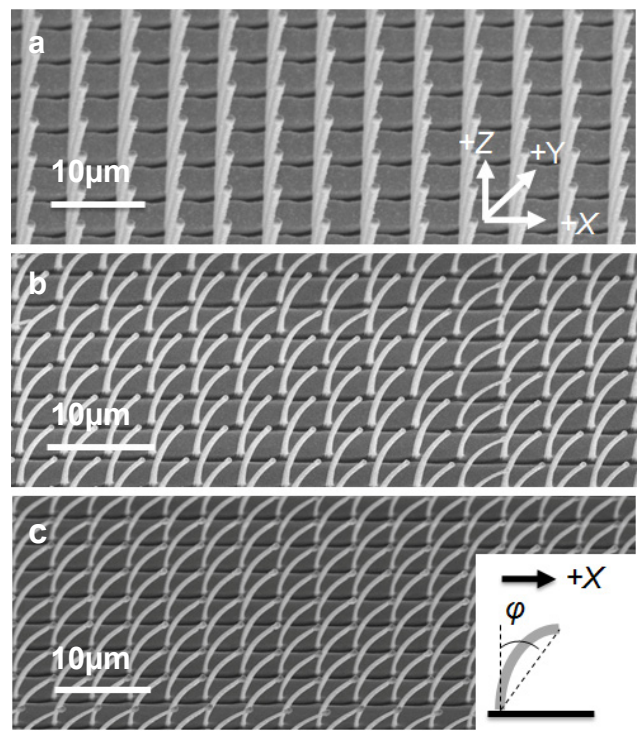

Figure 1: Scanning electron micrographs (SEMs) of fabricated asymmetric nanostructured surfaces with 500-750 $\mathrm{nm}$ pillars and deflection angles of a) $7^{\circ}$, b) $12^{\circ}$ and c) $25^{\circ}$. Schematic in inset defines the deflection angle, $\varphi$.

layer using an initiated chemical vapor deposition (iCVD) process $[9,10]$. The deposited polymer allowed controlled investigations of the effect of geometry on liquid spreading behavior. Different polymers were used in the experiments to tailor the surface energy of nanostructures, such that the effect of surface properties on spreading behavior could also be studied. In addition, the polymer served as the dielectric layer for electrowetting experiments.

\section{UNI-DIRECTIONAL SPREADING \\ Phenomenon}

When a water droplet is deposited on a typical periodic nanostructured hydrophilic surface, the droplet spreads in all directions (Fig. 2a). Asymmetric nanostructures, on the other hand, can introduce local energy barriers via geometry in different directions, so that in some cases, uni-directional spreading is achieved. For example, when the same droplet shown in Fig. 2a is placed on the deflected nanopillar array with a deflection angle of $12^{\circ}$, the liquid spreads only in the $+\mathrm{X}$ direction (Fig. 2b). Fig. 3 shows time-lapse images of the side and top view of such unidirectional spreading behavior. The images demonstrate that the liquid droplet propagates primarily in the $+X$ direction while the contact line pins in both the $-\mathrm{X}$ direction and along the $\mathrm{Y}$-axis. To investigate the spreading behavior further, we studied the liquid film propagating ahead of the droplet within the nanopillars, as indicated by the arrow in Fig. $3 \mathbf{b}$.

\section{Theory and Model}

We found that the behavior of the liquid film was wellcorrelated with the behavior of the macroscopic droplet, i.e. the liquid film propagation determines the direction of the 

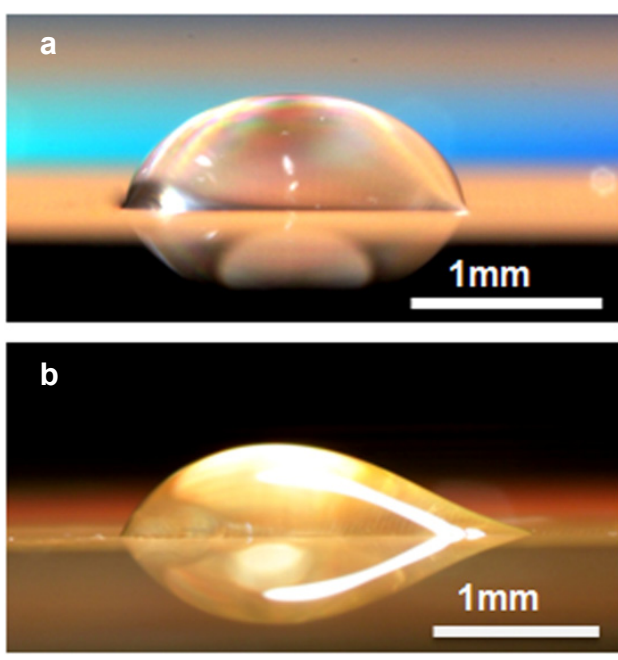

Figure 2: Spreading behaviors and droplet shapes on typical symmetric nanostructured surfaces and the asymmetric nanostructured surfaces fabricated in this work. a) $1 \mu \mathrm{L}$ water droplet symmetrically spreads on typical vertical nanopillars. b) The same droplet spreads uni-directionally on asymmetric nanostructures with a $12^{\circ}$ deflection angle. The scale bars in the insets are $10 \mu \mathrm{m}$.

macroscopic droplet spreading. To explain the uni-directional liquid film propagation, we developed a simple two-dimensional geometric model. The asymmetric pillars were simplified as slanted rectangles in the $2 \mathrm{D}$ model, as shown in the Fig. 4 . The model assumes that the liquid film propagate only if the contact line is able to reach the next row of pillars. In addition, when liquid film propagates, the local contact angle of the liquid cannot be smaller than $\theta_{e q}$ according to Young's Equation [5] due to the fact that $\theta_{e q}$ is the lowest energy state. Therefore, $\theta_{e q}$ of the liquid must be equal to or smaller than a critical angle defined as $\theta_{c r}$ shown in the Fig. 4. Because the film propagates at the height of the pillars, then $\theta_{c r}=\tan ^{-1}\left(H_{\text {eff }} l_{\text {eff }}\right)$, where $H_{\text {eff }}$ and $l_{\text {eff }}$ are the effective height of the pillars and spacing between pillars, respectively. Thus, the critical angle in the $+\mathrm{X}$ direction is given by

$$
\theta_{c r,+x}=\tan ^{-1} \frac{(H / l) \cos \varphi}{1-(H / l) \sin \varphi}
$$

where $H$ and $l$ are the actual height and spacing between the pillars, respectively, and $\varphi$ is the pillar deflection angle. Similarly, for the case of liquid film propagation in the $-\mathrm{X}$ direction, the critical angle is given by

$$
\theta_{c r,-x}=\tan ^{-1} \frac{(H / l) \cos \varphi}{1+(H / l) \sin \varphi}
$$

If $\theta_{c r}>\theta_{e q}$, then the liquid film propagates within the nanopillars, otherwise, the liquid film pins.

To study the spreading behavior and validate the theoretical model, a series of experiments were conducted. We used DI water and DI water with surfactants (Triton X-100 with concentrations ranging from $0.01 \%$ to $0.00125 \%$ by volume) on the polymer (poly(cycohexyl methacrylate-co-divinyl benzene)) coated surface with an equilibrium contact angle of $80^{\circ}$ (DI water). On this surface, surfactants were necessary to decrease the equilibrium contact angle in order to satisfy the condition for imbibition [11], which is the condition in which the liquid can start to spread. a

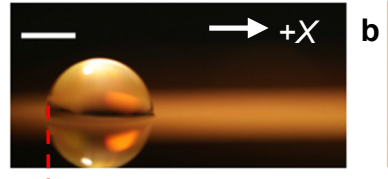

I
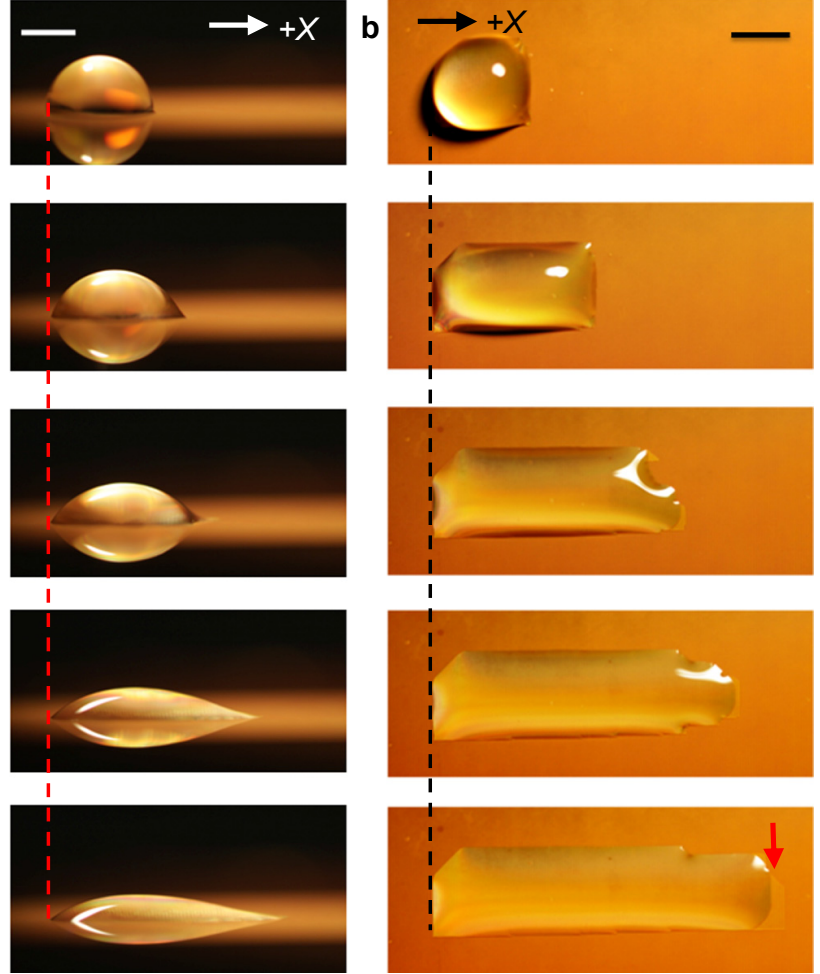

I
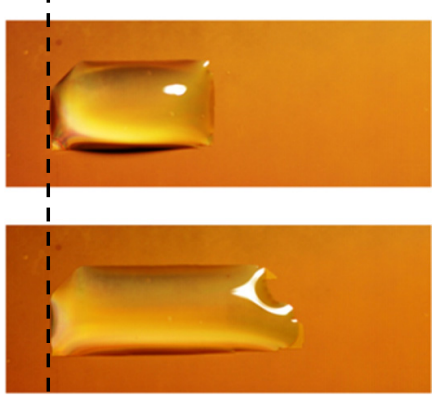

I
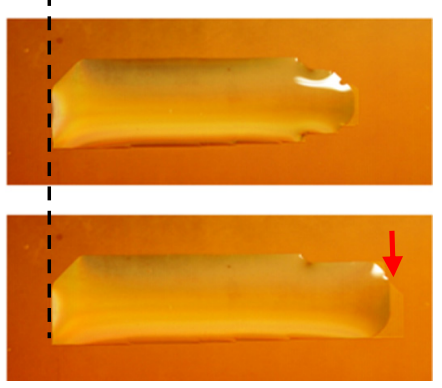

Figure 3: Time-lapse images of uni-directional spreading of a liquid droplet. a) Side view and b) top view of a $1 \mu \mathrm{L}$ droplet of DI water with $0.002 \%$ by volume of surfactant spreading on a surface with pillar diameters of 500-750 $\mathrm{nm}$, spacings of $3.5 \mu \mathrm{m}$, and heights of $10 \mu \mathrm{m}$ deflected at $12^{\circ}$ as shown in Fig. 2. The initial location of the droplet contact line in the $-X$ direction is indicated by the dotted lines, where the contact line stays pinned throughout the spreading process. The liquid film propagates in front of the macroscopic droplet as highlighted by the arrow in $\boldsymbol{b}$. The scale bars are $1 \mathrm{~mm}$.
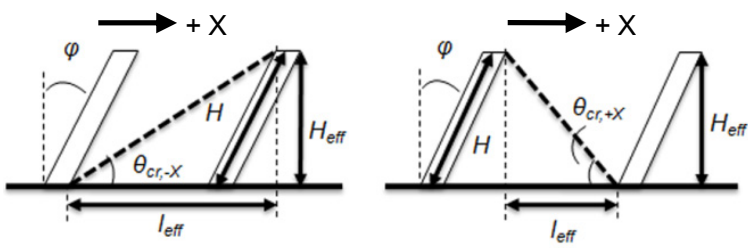

Figure 4: Schematics explaining the geometries for the developed model that determines the critical angle, $\theta_{c r-x}$ and $\theta_{c r+x}$ to initiate spreading in both $-X$ (top) and $+X$ (bottom), respectively. The

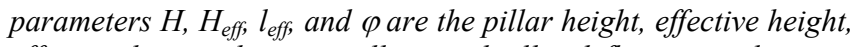
effective distance between pillars, and pillar deflection angle.

Fig. 5 depicts the experimental results of uni-directional spreading and the theoretical curves based on the geometric model. The model is plotted for the case $\theta_{c r}=\theta_{e q}$ in both $+\mathrm{X}$ and $-\mathrm{X}$ where the $H / l$ ratio is $2.87(H=10 \mu \mathrm{m})$ for different pillar deflection angles and equilibrium contact angles. The region bounded by $\theta_{c r,-x}$ and $\theta_{e q}=65^{\circ}$ (i.e., the imbibition condition) indicates the parameter space (in blue) that allows uni-directional spreading. The square symbols $(\square)$ indicate experiments in which the liquid film propagates only in the $+X$ direction, whereas the circles (O) are experiments where the film propagates directionally (in $+\mathrm{X}$ and $-\mathrm{X})$. The triangles $(\Delta)$ show instances where the spreading is 
nearly uni-directional, i.e., the film propagates in $-\mathrm{X}$ but the propagation rate was at least five times slower than in $+X$. The crosses $(\times)$ represent experiments where the film does not propagate at all due to the imbibition condition.

\section{CONTROL OF SPREADING BEHAVIOR}

In the previous studies, we tailored the polymers to ensure that the imbibition condition can be satisfied to achieve unidirectional spreading [8]. In the subsequent step of this work, we studied the effect of an applied electric potential across the droplet and the surface, to achieve on-demand control of spreading directionalities. Electrowetting is a well-known phenomenon that involves modifying the wettability and the contact angle of a liquid (electrolyte) on the surface using an electric potential $[2,12,13]$. The effect due to electrowetting on contact angle based on a thermodynamic model is given by [13]

$$
\theta=\cos ^{-1}\left(\cos \theta_{e q}+\frac{\varepsilon_{0} \varepsilon_{d}}{2 d \gamma_{l v}} V^{2}\right)
$$

where $\theta$ is the contact angle under electrowetting effect, $\varepsilon_{0}$ is vacuum permittivity, $\varepsilon_{d}$ is relative permittivity of the dielectric layer, $d$ is the thickness of the dielectric layer, $\gamma_{l v}$ is surface tension of the liquid, and $V$ is the applied electric voltage. In the experiments, we used $0.1 \mathrm{M} \mathrm{NaCl}$ solution on the surfaces coated with another polymer (poly ( $\mathrm{N}$-isopropylacrylamide-co-di(ethylene glycol) divinylether)). The liquid ( $\mathrm{NaCl}$ solution) on the polymer coated surfaces has an equilibrium contact angle, $\theta_{e q}$, of $66^{\circ}$ and the contact angle with electrowetting can be calculated based on equation (3). However, to predict $\theta$, the relative permittivity of the polymer, $\varepsilon_{d}$, needs to be determined, which is dependent on the frequency of the applied voltage. In this work, we used experiments to determine the relationship between the droplet contact angle and applied voltage, $V$, as shown in Fig. 6.

A droplet of $0.1 \mathrm{M} \mathrm{NaCl}$ solution deposited on asymmetric nanostructured surfaces initially remains static due to the imbibition condition. With electrowetting, the contact angle of the liquid decreases, and the liquid starts to spread when the imbibition condition is satisfied. In the presence of asymmetric nanostructures, when the applied potential is sufficient to satisfy the imbibition condition, the liquid will spread uni-directionally as shown in Fig. 7a. An example case is shown with an applied voltage of $V=1.5 \mathrm{~V}$, which causes the initially static droplet to spread in only the $+X$, direction (Fig. 7b). Furthermore, by changing the magnitude of the electric potential, different droplet spreading directionalities can be achieved. As the voltage is increased to $V=2.1 \mathrm{~V}$, the liquid unpins in $-\mathrm{X}$ and spreads bidirectionally. The spreading, however, is asymmetric; the rate is three times faster in $+X$ as compared to $-X$ (Fig. 7c). Moreover, with increasing applied $V$, the asymmetry decreases. In the case of an applied $V>2.5 \mathrm{~V}$, the liquid spreading is nearly symmetric, i.e. the rates in $+\mathrm{X}$ and $-\mathrm{X}$ are approximately equal (Fig. 7d).

We further conducted a series of experiments with different pillar deflection angles and the contact angles, $\theta$, associated with the applied potentials. The experimental results is also overlaid with the model for uni-directional spreading and plotted in Fig. 8 for the case $\theta_{c r}=\theta$ where the $H / l$ ratio is 2.57 . In Fig. 8 , the square symbols $(\square)$ indicate experiments in which the liquid propagates only in the $+X$ direction. The triangles $(\Delta)$ shows the spreading is bi-directional asymmetric, while circles ( $\circ$ ) show experimental results of nearly bi-directional symmetric liquid spreading. The crosses $(\times)$ represent experiments where the liquid does not spread at all due to the imbibition condition.

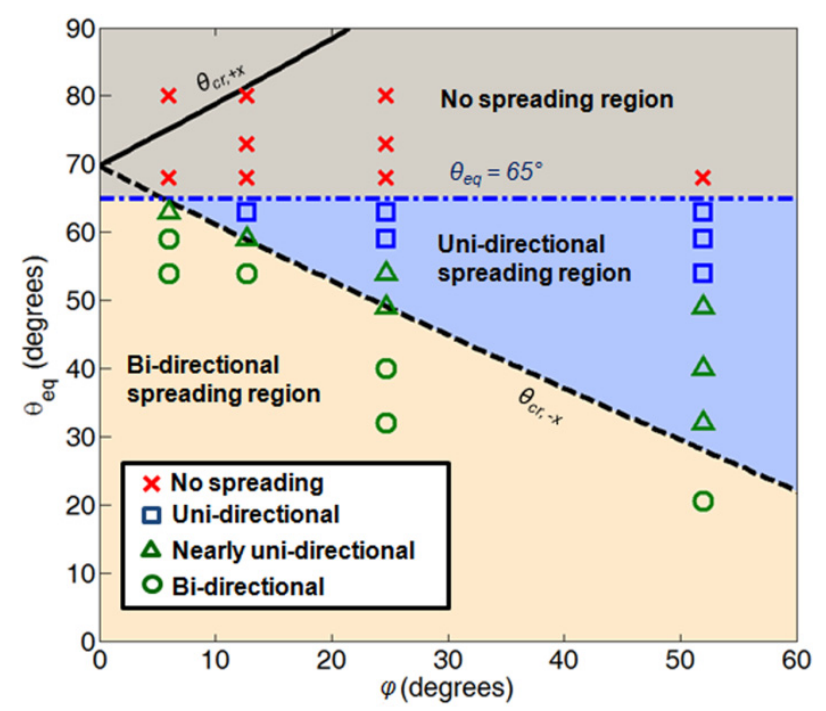

Figure 5: Experimental results and the theoretical curves predicting uni-directional liquid spreading. The squares ( $\square$ ), circles $(\circ)$, triangles $(\Delta)$, and crosses $(\times)$ show experimental results of uni-directional, bi-directional, nearly uni-directional, and no liquid propagation, respectively. The theoretical curves based on the proposed model are shown for $\theta_{e q}=\theta_{c r}$ with varying deflection angles, $\varphi$, for $H / l=2.87$. The center region (blue) bound by $\theta_{c r,-x}$ and $\theta_{\text {eq }}=65^{\circ}$ represents the parameter space that leads to uni-directional liquid spreading.

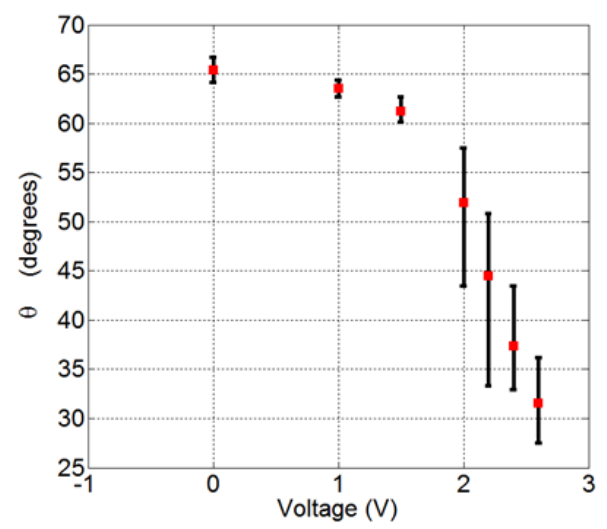

Figure 6: Relationship between the contact angles of the droplet, $\theta$, with varying applied voltage, $V$.

Despite the simplifications in the proposed model, the experiments and model show excellent agreement. The proposed 2-D model can well predict spreading behaviors because the liquid equilibrates along the $\mathrm{Y}$-axis faster than along the $\mathrm{X}$-axis thereby causing liquid propagation primarily along the $\mathrm{X}$-axis for these fabricated geometries.

\section{CONCLUSION}

Asymmetric nanostructured surfaces were fabricated that can achieve uni-directional liquid spreading. With electrowetting, various spreading behaviors on the nanostructured surfaces have been demonstrated. A combined study of experiments and modeling shows the effect of nanopillar geometry and surface energy on spreading behavior. The study provides design guidelines to tune the droplet behavior from uni-directional to 
a
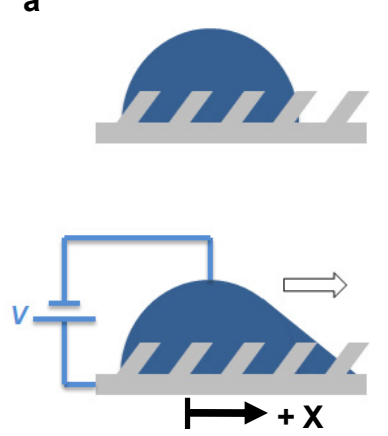
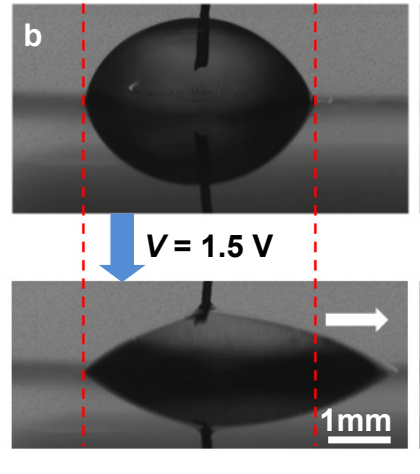
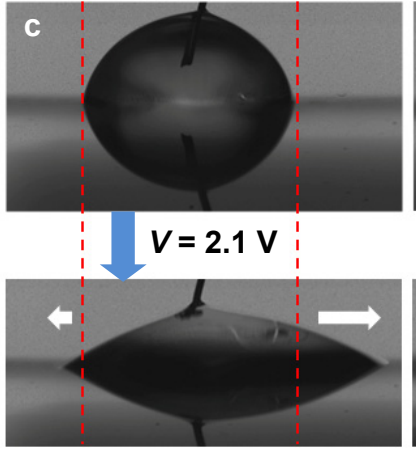
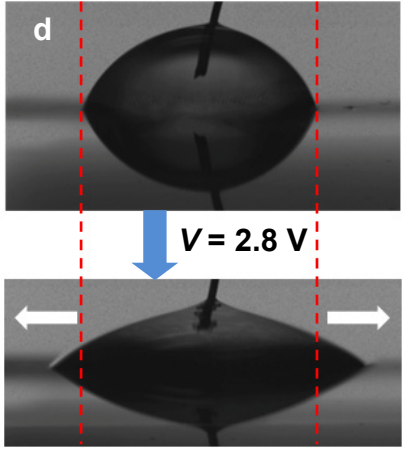

Figure 7: Manipulation of spreading behavior with water droplets on asymmetric nanostructured surfaces by electrowetting. a) Schematic shows experimental setup where an initially static symmetric droplet spreads only to the right ( $+X$ direction) with an applied potential $V$, which represents results shown in b). b) With $V=1.5 \mathrm{~V}$, the droplet spreads uni-directionally $($ in $+X)$. c) With V=2.1 V, the droplet spreads bi-directionally (in $+X$ and $-X$ ), but asymmetrically where the rate is at least three times faster in $+X$ than in $-X$. $d)$ With $V=2.8 V$, the droplet spreads bi-directionally, but the rates are comparable in $+X$ and $-X$. The red dotted lines highlight the initial positions of the droplets on both sides, and the white arrows highlight the spreading rates and directions.

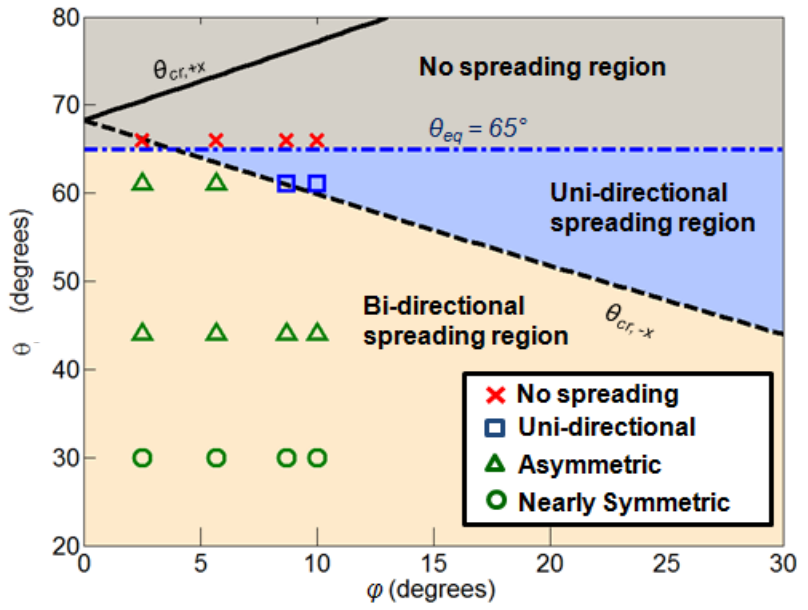

Figure 8: Experimental results overlaid with model predictions for different deflection angles, $\varphi$, for $H / l=2.57$, and contact angles, $\theta$, obtained from the applied voltages, $V$. The crosses $(\times)$, squares $(\square)$, triangles $(\Delta)$, and circles $(\circ)$ show experimental results of no spreading, uni-directional, bi-directional asymmetric, and nearly bi-directional symmetric liquid spreading, respectively.

bi-directional asymmetric or symmetric spreading using both nanostructure design and applied electric fields. These results suggest exciting opportunities to achieve real-time control of wetting behavior.

\section{ACKNOWLEDGEMENT}

The authors gratefully acknowledge funding support from the National Science Foundation (under Award EEC-0824328), the DARPA Young Faculty Award, and the Northrop Grumman New Faculty Innovation Grant. The authors would also like to acknowledge the Intel Higher Education Grant for a generous computer donation, and the MIT Microsystems Technology Lab.

\section{REFERENCES}

[1] D Quéré, "Non-sticking Drops", Rep. Prog. Phys., 68, 24952532 (2005).

[2] T. N. Krupenkin, J. A. Taylor, T. M. Schneider, and S. Yang, "From Rolling Ball to Complete Wetting: The Dynamic
Tuning of Liquids on Nanostructured Surfaces" Langmuir, 20, 3824-3827 (2004).

[3] C. W. Extrand, S. I. Moon, P. Hall, and D. Schmidt, "Superwetting of Structured Surfaces", Langmuir, 23, 88828890 (2007).

[4] R. Xiao, K.-H. Chu, and E. N. Wang, "Multilayer Liquid Spreading on Superhydrophilic Nanostructured Surfaces", Appl. Phys. Lett., 94, 193104 (2009).

[5] L. Courbin, E. Denieul, E. Dressaire, M. Roper, A. Ajdari, and H. A. Stone "Imbibition by polygonal spreading on microdecorated surfaces", Nature Mater., 6, 661-664 (2007).

[6] J. Y. Chung, J. P. Youngblood, and C. M. Stafford, "Anisotropic Wetting on Tunable Micro-wrinkled Surfaces", Soft Matter, 3, 1163-1169 (2007).

[7] J. Drelich, J. L. Wilbur, J. D. Miller, and G. M. Whitesides, "Contact Angles for Liquid Drops at a Model Heterogeneous Surface Consisting of Alternating and Parallel Hydrophobic Hydrophilic Strips", Langmuir, 12, 1913-1922 (1996).

[8] K.-H. Chu, R. Xiao, and E. N. Wang, "Uni-directional liquid spreading on asymmetric nanostructured surfaces", Nature Mater., accepted (2010)

[9] S.H. Baxamusa, S.G. Im, and K.K. Gleason, "Initiated and oxidative chemical vapor deposition: a scalable method for conformal and functional polymer films on real substrates" PCCP, 11, 5227-5240 (2009).

[10] M.E. Alf, A. Asatekin, M.C. Barr, S.H. Baxamusa, H. Chelawat, G. Ozaydin-Ince, C.D. Petruczok, R. Sreenivasan, W.E. Tenhaeff, N.J. Trujillo, S. Vaddiraju, J. Xu, and K.K. Gleason. "Chemical Vapor Deposition (CVD) of conformal, functional, and responsive polymer films”, Adv. Mater., 21, 1-35 (2009).

[11] J. Bico, C. Tordeux, and D. Quéré, "Rough Wetting", Europhys. Lett., 55, 214-220 (1999).

[12] E. N. Wang, M.A. Bucaro, J.A. Taylor, P. Kolodner, J. Aizenberg, and T. Krupenkin, "Droplet mixing using electrically tunable superhydrophobic nanostructured surfaces", Microfluid Nanofluid, 7, 137-140 (2009).

[13] F. Mugele, and J.-C. Baret, "Electrowetting: from basic to applications”, J. Phys., 17, R705-R774 (2005).

\section{CONTACT}

*K.-H. Chu, tel: +1-617-253-2198; khchu@mit.edu 